\title{
A novel in vitro analog expressing learning-induced cellular correlates in distinct neural circuits
}

\author{
Harris A. Weisz, ${ }^{1,2}$ Marcy L. Wainwright, ${ }^{1}$ and Riccardo Mozzachiodi ${ }^{1}$ \\ ${ }^{1}$ Department of Life Sciences, Texas A\&M University - Corpus Christi, Corpus Christi, Texas 78412, USA
}

\begin{abstract}
When presented with noxious stimuli, Aplysia exhibits concurrent sensitization of defensive responses, such as the tailinduced siphon withdrawal reflex (TSWR) and suppression of feeding. At the cellular level, sensitization of the TSWR is accompanied by an increase in the excitability of the tail sensory neurons (TSNs) that elicit the reflex, whereas feeding suppression is accompanied by decreased excitability of $\mathrm{B} 51$, a decision-making neuron in the feeding neural circuit. The goal of this study was to develop an in vitro analog coexpressing the above cellular correlates. We used a reduced preparation consisting of buccal, cerebral, and pleural-pedal ganglia, which contain the neural circuits controlling feeding and the TSWR, respectively. Sensitizing stimuli were delivered in vitro by electrical stimulation of afferent nerves. When trained with sensitizing stimuli, the in vitro analog expressed concomitant increased excitability in TSNs and decreased excitability in B51, which are consistent with the occurrence of sensitization and feeding suppression induced by in vivo training. This in vitro analog expressed both short-term (15 min) and long-term (24 h) excitability changes in TSNs and B51, depending on the amount of training administered. Finally, in vitro application of serotonin increased TSN excitability without altering B51 excitability, mirroring the in vivo application of the monoamine that induces sensitization, but not feeding suppression.
\end{abstract}

In the past decades, neuroscientists have greatly benefited from the development of in vitro reduced preparations, obtained from isolated portions of the central and peripheral nervous system of vertebrates and invertebrates, and capable of expressing features of brain functions exhibited in vivo (Kovac and Davis 1977; Lukowiak and Sahley 1981; Hawkins et al. 1983; Byrne 1987; Zhang et al. 1994; Nargeot et al. 1997; Proekt et al. 2004; Marder et al. 2005; Mentel et al. 2008; Keifer and Zheng 2010; Mozzachiodi and Byrne 2010; Crossley et al. 2016). These preparations, commonly termed "in vitro analogs" have been particularly useful to investigate the cellular and molecular mechanisms of learning and memory in identified neural circuits (Hawkins et al. 1983; Walters and Byrne 1983; Kemenes et al. 1997; Nargeot et al. 1997; Burrell et al. 2001; Scuri et al. 2002; Keifer and Zheng 2010).

Several in vitro analogs have been developed using the nervous system of the marine mollusk Aplysia to analyze the mechanisms of various forms of learning, including habituation (Frost et al. 1997) and sensitization (Zhang et al. 1994; Antonov et al. 1999; Marinesco and Carew 2002; Philips et al. 2011), both aversive and appetitive forms of classical conditioning (Walters and Byrne 1983; Antonov et al. 2001; Mozzachiodi et al. 2003; Lorenzetti et al. 2006) and appetitive operant conditioning (Nargeot et al. 1999a,b; Mozzachiodi et al. 2008; Sieling et al. 2014). The use of these in vitro analogs has been crucial for characterizing learning-dependent mechanisms because it allows one to conduct experiments that are not otherwise feasible in vivo, such as manipulation of the mechanisms responsible for cellular changes at the level of both neural circuits and single cells (Nargeot et al. 1999b; Antonov et al. 2003, 2007; Mozzachiodi et al. 2008, 2013; Sieling et al. 2014). Up to now, however, one limitation presented by in vitro analogs of learning is that their use has been primarily restricted to the study of individual circuits, examined one at a

\footnotetext{
${ }^{2}$ Present address: Department of Anesthesiology, The University of Texas Medical Branch, Galveston, TX 77555, USA.

Corresponding author: riccardo.mozzachiodi@tamucc.edu

Article is online at http://www.learnmem.org/cgi/doi/10.1101/lm.045229.117.
}

time (Walters and Byrne 1983; Zhang et al. 1994; Nargeot et al. 1997; Mozzachiodi et al. 2003; Philips et al. 2011). The goal of this study was to expand upon previously established in vitro analogs and develop one capable of coexpressing learning-dependent changes in distinct neural circuits and in different temporal domains.

Recent work from our laboratory revealed that, in Aplysia, noxious electrical stimuli produce two concurrent behavioral changes: a well-characterized enhancement of defensive withdrawal reflexes, known as sensitization (for review, see Kandel 2001; Byrne and Hawkins 2015), and suppression of feeding behavior (Acheampong et al. 2012; Shields-Johnson et al. 2013). The above behavioral changes share analogous time courses that depend on the amount of aversive stimulation (Acheampong et al. 2012; Shields-Johnson et al. 2013). Training consisting of a single-trial produces sensitization of the tail-induced siphon withdrawal reflex (TSWR) and suppression of feeding at $15 \mathrm{~min}$, but not $24 \mathrm{~h}$ after training (Acheampong et al. 2012). Training consisting of four trials induces sensitization and feeding suppression lasting at least $24 \mathrm{~h}$ (Acheampong et al. 2012; Shields-Johnson et al. 2013).

Cellular correlates of sensitization and feeding suppression have been previously identified in the corresponding underlying neural circuits. Within the neural circuit controlling the TSWR, plasticity associated with sensitization has been identified at the level of the sensory neurons in the ventrocaudal (VC) cluster of the pleural ganglion that respond to tail stimulation (Walters et al. 1983a,b, 2004). These tail sensory neurons (TSNs) make monosynaptic contacts onto motor neurons in the pedal ganglion, generating contraction of the tail, and polysynaptic contacts onto motor neurons in the abdominal ganglion, generating contraction

(C) 2017 Weisz et al. This article is distributed exclusively by Cold Spring Harbor Laboratory Press for the first 12 months after the full-issue publication date (see http://learnmem.cshlp.org/site/misc/terms.xhtml). After 12 months, it is available under a Creative Commons License (AttributionNonCommercial 4.0 International), as described at http://creativecommons. org/licenses/by-nc/4.0/. 
In vitro learning correlates in multiple circuits

of the siphon (Cleary et al. 1995). Training with sensitizing stimuli increases the excitability of the TSNs both in the short and long term (Zhang et al. 1994; Cleary et al. 1998; Liao et al. 1999; Marinesco and Carew 2002). In Aplysia, serotonin $(5-\mathrm{HT})$ mediates sensitization and its cellular correlates at the level of the sensory-to-motor neuron synapses and can serve as analog of sensitization training (Walters et al. 1983b; Byrne and Kandel 1996; Byrne and Hawkins 2015). In particular, 5-HT treatment increases TSN excitability in an intrinsic manner, as demonstrated by results obtained both in neurons from surgically excised sensory clusters (Baxter and Byrne 1990) and in neurons isolated in culture (Dale et al. 1987; Liu et al. 2011).

Within the neural circuit controlling feeding, sensitization training decreases the excitability of neuron B51 (ShieldsJohnson et al. 2013; Hernandez et al. 2017). B51 is a premotor neuron that contributes to the genesis of ingestive buccal motor programs, which are in vitro neurophysiological correlates of a bite/swallow (Plummer and Kirk 1990; Nargeot et al. 1999a,b; Cropper et al. 2004; Sasaki et al. 2013). B51 expresses an intrinsically generated all-or-nothing burst of action potentials (i.e., plateau potential; Plummer and Kirk 1990) that participate in the selection of ingestive buccal motor programs (Nargeot et al. 1999a,b; Brembs et al. 2002; Nargeot and Simmers 2012). Notably, B51 exhibits in vitro decision-making features that enable it to switch in an all-or-nothing fashion from an inactive subthreshold state to an active plateau potential during the occurrence of ingestive buccal motor programs (Nargeot and Simmers 2012; Mozzachiodi et al. 2013; Dickinson et al. 2015). The decrease of B51 excitability induced by in vivo sensitization training is not accompanied by changes in input resistance, resting membrane potential, or synaptic input (ShieldsJohnson et al. 2013) and persists when synaptic transmission is pharmacologically blocked (Hernandez et al. 2017).

The above findings provided the framework for the development of an in vitro analog coexpressing excitability changes in TSNs and B51 similar to those induced by in vivo sensitization training. We implemented a previously established in vitro analog that expresses cellular correlates of sensitization in the isolated pleural-pedal ganglia, including increased TSN excitability and facilitation of the sensorimotor neuron synapses (Zhang et al. 1994; Liao et al. 1999; Marinesco and Carew 2002). As an in vitro representation of sensitizing stimuli, we used patterns of simultaneous electrical stimulation of afferent pedal nerves P8 and P9 because they induce cellular correlates of sensitization in the isolated pleural-pedal ganglia (Goldsmith and Byrne 1989; Zhang et al. 1994; Liao et al. 1999; see Materials and Methods for details). In order to retain the critical components of the neural circuits controlling the TSWR and feeding and to capture the learning-induced changes occurring within these circuits, the buccal, cerebral, and pleural-pedal ganglia were isolated in vitro together with their interganglionic connectives (Fig. 1A; Zhang et al. 1994; Cleary et al. 1995; Cropper et al. 2004; Marinesco et al. 2004). We used increase of TSN excitability and decrease of B51 excitability as readouts of sensitization and feeding suppression, respectively (Cleary et al. 1998; Shields-Johnson et al. 2013).

\section{Results}

\section{The in vitro analog coexpressed short-term excitability changes in TSNs and B51}

The first goal of this study was to examine whether short-term changes in the excitability of TSNs and B51 were concurrently induced by in vitro sensitization training. For the in vitro training, we modified a previously established protocol, consisting of four 10 -sec trains of electrical stimuli applied simultaneously to both pedal nerves P8 and P9 (Fig. 1B2; see Materials and Methods for details), which induces synaptic facilitation lasting at least $24 \mathrm{~h}$ (Zhang et al. 1994). From this paradigm, we derived a training protocol, comprised of a single train of P8/P9 electrical stimulation (Fig. 1B1), which is analogous to an in vivo paradigm that produces sensitization and suppression of feeding lasting for at least $15 \mathrm{~min}$ (Acheampong et al. 2012). We initially tested whether the singletrial in vitro training induced short-term (15 min) increased TSN excitability in the isolated pleural-pedal ganglia. TSN excitability was measured as the number of action potentials evoked by a 1-sec, 2-nA current injection (Cleary et al. 1998). Fifteen minutes after training, TSN excitability was significantly increased in trained ganglia (change in number of spikes: $180.16 \pm 85.10 \%$ of pretest; $n=7$ ) when compared with untrained ganglia (change in number of spikes: $0.44 \pm 16.10 \%$ of pretest; $n=9 ; P<0.05 ; U=$ 9.50; Mann-Whitney $U$-test). There was no significant effect of training on the resting membrane potential $\left(V_{\mathrm{m}}\right)$ of TSNs (change in $V_{\mathrm{m}}$, trained: $5.25 \pm 3.50 \%$ of pretest; $n=7$; untrained: $8.13 \pm$ $2.47 \%$ of pretest; $n=9 ; \quad P=0.75 ; \quad U=28.00$; Mann-Whitney $U$-test). These results indicate that P8/P9 electrical stimulation successfully served as in vitro sensitization training.

We next tested the hypothesis that the single-trial in vitro training induced concurrent excitability changes in both TSNs and B51. The analog configuration consisted of the buccal, 
cerebral, and pleural-pedal ganglia (Fig. 1A). B51 excitability was measured as the current threshold to elicit the regenerative burst of action potentials known as a plateau potential (Nargeot et al. 1999a). Fifteen minutes after training (Fig. 1B1), we observed the coexpression of increased TSN excitability (Fig. 2A1,A2) and decreased B51 excitability (Fig. 2B1,B2). The above excitability changes were not accompanied by modifications of $V_{\mathrm{m}}$ in TSNs, or by modifications of $V_{\mathrm{m}}$ or input resistance $\left(R_{\mathrm{m}}\right)$ in B51 (Table $1)$. These findings indicate that the in vitro analog successfully coexpressed short-term changes in intrinsic excitability in both TSNs and B51. In 77\% of the preparations examined, B51 exhibited inhibitory synaptic potentials (IPSPs) originating from presynaptic neurons within the feeing neural circuit, including B52 (Plummer and Kirk 1990; Nargeot et al. 2002). Changes in this inhibitory synaptic input might potentially contribute to the training-induced decrease of B51 excitability. To address this issue, we examined the magnitude of the IPSPs in the trained preparations before and after training (see Materials and Methods for details). The average IPSP amplitude did not change after the single-trial in vitro training (pretest: $-2.05 \pm 0.46 \mathrm{mV}$; post-test: $-2.61 \pm 0.73 \mathrm{mV}, n=7 ; P=0.69 ; W=-6.00 ;$ Wilcoxon signed rank test).

\section{In vitro training did not alter the excitability of neuron $B 4 / 5$}

In order to test whether learning-dependent changes were limited to predicted sites of plasticity, such as B51 and TSNs, in the second
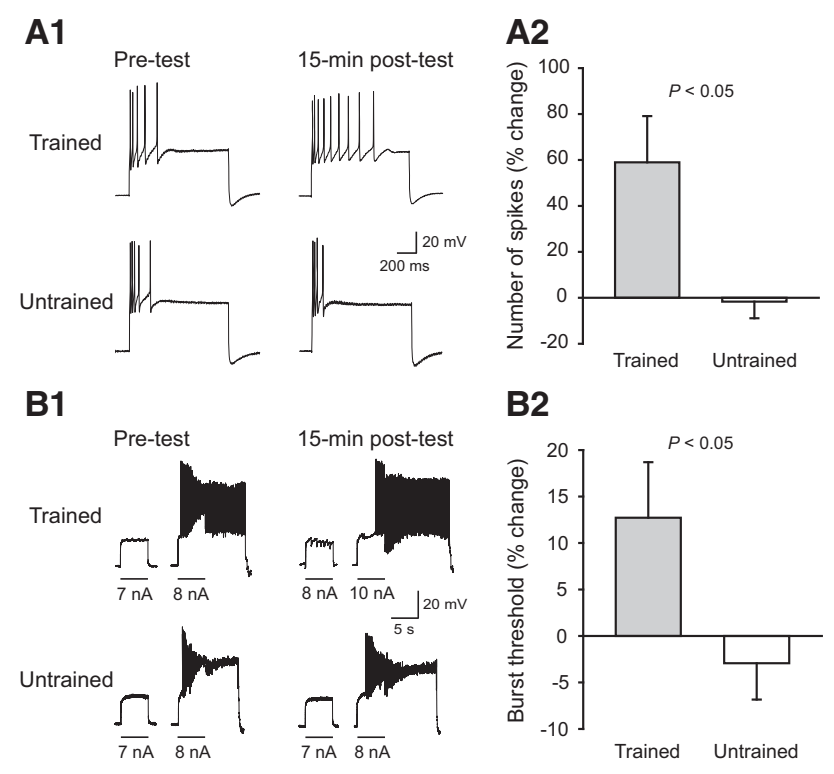

Figure 2. Single-trial in vitro training induced a concurrent increase of TSN excitability and a decrease of B51 excitability $15 \mathrm{~min}$ after training. (A1) Sample traces of TSN firing from trained and untrained preparations. (A2) Summary data illustrate that the single-trial in vitro training significantly increased TSN excitability (change in number of spikes, trained: $58.97 \pm 20.14 \%$ of pretest; $n=13$; untrained: $-1.68 \pm 7.22 \%$ of pretest; $n=10 ; P<0.05 ; U=21.00$; Mann-Whitney $U$-test). (B1) Sample traces of B51 burst threshold from trained and untrained preparations. (B2) Summary data illustrate that the single-trial in vitro training significantly increased B51 burst threshold (change in burst threshold, trained: $12.72 \pm$ $5.98 \%$ of pretest; $n=10$; untrained: $-2.93 \pm 3.92 \%$ of pretest; $n=9 ; P<$ 0.05; $U=20.00$; Mann-Whitney $U$-test). In these and the following figures, values are expressed as mean \pm SEM. experiment, we used the above in vitro analog to analyze the effects of in vitro training on the membrane properties of another neuron in the feeding neural circuit: B4/5. B4/5 are two pairs of multifunctional neurons, which are involved in the neural mechanisms of feeding motor program switching (Gardner 1977; Church and Lloyd 1994; Warman and Chiel 1995; Jing and Weiss 2001). Based on previous negative results observed following appetitive classical conditioning (Lechner et al. 2000; Mozzachiodi et al. 2003), we hypothesized that B4/5 would not be a site of plasticity following sensitization training. B4/5 excitability was measured as the number of action potentials evoked by a 1-sec, $10-n A$ current injection. Training procedures were identical to those used in the previous experiment. Although the single-trial in vitro training successfully increased the excitability of TSNs 15 min after training (Fig. 3A1,A2), it did not alter the excitability of $\mathrm{B} 4 / 5$ (Fig. 3B1,B2). The single-trial in vitro training also did not change the $V_{\mathrm{m}}$, or the $R_{\mathrm{m}}$ of $\mathrm{B} 4 / 5$ (Table 1 ).

\section{The in vitro analog coexpressed long-term excitability changes in TSNs and B51}

In the next experiment, we investigated whether the short-term excitability changes observed in TSNs and B51 could be extended to the long term $(24 \mathrm{~h})$. Previous work in Aplysia indicates that training with repeated trials of noxious stimuli induces concurrent sensitization and feeding suppression lasting at least $24 \mathrm{~h}$ (Acheampong et al. 2012; Shields-Johnson et al. 2013). Therefore, we tested whether multiple in vitro sensitization trials induced concomitant long-term excitability changes in TSNs and B51 persisting for at least $24 \mathrm{~h}$. We used a previously developed in vitro training protocol, consisting of four trials (each identical to that used in the short-term protocol described above) spaced $30 \mathrm{~min}$ apart (Fig. 1B2; Goldsmith and Byrne 1989; Zhang et al. 1994). This in vitro training significantly increased the excitability of TSNs (Fig. 4A1,A2) and decreased the excitability of B51 (Fig. $4 \mathrm{~B} 1, \mathrm{~B} 2)$ at the 24 -h post-test. Similar to the results obtained with the single-trial in vitro training, the above long-term excitability changes were not accompanied by modifications of resting membrane properties in either TSNs, or B51 (Table 1). Moreover, the analysis of inhibitory synaptic input to B51 revealed that the average IPSP amplitude did not change after the four-trial in vitro training (pretest: $-3.93 \pm 1.09 \mathrm{mV}$; post-test: $-3.37 \pm 0.72 \mathrm{mV}, n$ $=8 ; P=0.74 ; W=-6.00$; Wilcoxon signed rank test). These findings indicate that cellular correlates of sensitization and feeding suppression are coexpressed $24 \mathrm{~h}$ following the four-trial in vitro training.

\section{Serotonin differentially modulated the excitability of TSNs and B51}

In the final experiment, we characterized the effects of in vitro application of serotonin (5-HT) on the excitability of TSNs and B51. Previous work in Aplysia indicates that 5-HT mediates sensitization and its underlying cellular underpinnings, including increased TSN excitability (Walters et al. 1983a,b; Kandel 2001; Byrne and Hawkins 2015). However, an in vivo 5-HT bath-application protocol, which induced sensitization of the TSWR, did not induce feeding suppression or changes in B51 excitability (Shields-Johnson et al. 2013). In this experiment, we analyzed whether the differential effects of 5-HT induced by in vivo 5-HT bath application were reproduced in vitro following 5 -HT treatment. We used the in vitro configuration in Figure 1A and the testing protocol of Figure 1B1 in which 5-min application of $50 \mu \mathrm{M} 5$-HT replaced P8/P9 electrical stimulation (Philips et al. 2011). 5-HT application significantly increased the excitability of TSNs 15 min after treatment (Fig. 5A1, A2), but did not alter B51 excitability (Fig. 5B1,B2). There was no 
Table 1. Effects of in vitro training protocols and 5-HT application on the resting properties of TSN, B51 and B4/5

\begin{tabular}{|c|c|c|c|c|c|c|c|}
\hline In vitro protocol & Neuron & Preparations $(n)$ & Resting property & Trained/5-HT & Untrained/ASW & $P$ & $U$ \\
\hline \multirow[t]{3}{*}{ Single-trial training } & TSN & Trained $=13 ;$ Untrained $=9$ & $V_{\mathrm{m}}$ & $-3.02 \pm 3.16 \%$ & $2.66 \pm 3.73 \%$ & 0.24 & 45.50 \\
\hline & B51 & Trained $=12 ;$ Untrained $=9$ & $V_{\mathrm{m}}$ & $6.96 \pm 1.92 \%$ & $6.70 \pm 1.32 \%$ & 0.62 & 46.50 \\
\hline & & Trained $=12 ;$ Untrained $=9$ & $R_{\mathrm{m}}$ & $1.24 \pm 4.74 \%$ & $-5.50 \pm 1.84 \%$ & 0.41 & 42.00 \\
\hline \multirow[t]{3}{*}{ Single-trial training } & TSN & Trained $=28 ;$ Untrained $=29$ & $V_{\mathrm{m}}$ & $-2.32 \pm 2.10 \%$ & $2.53 \pm 2.47 \%$ & 0.10 & 300.00 \\
\hline & B4/5 & Trained $=34 ;$ Untrained $=35$ & $V_{\mathrm{m}}$ & $0.73 \pm 1.56 \%$ & $3.37 \pm 1.28 \%$ & 0.41 & 526.00 \\
\hline & & Trained $=32 ;$ Untrained $=34$ & $R_{\mathrm{m}}$ & $0.04 \pm 3.06 \%$ & $2.10 \pm 1.92 \%$ & 0.88 & 532.00 \\
\hline \multirow[t]{3}{*}{ Four-trial training } & TSN & Trained $=14 ;$ Untrained $=17$ & $V_{\mathrm{m}}$ & $3.45 \pm 4.50 \%$ & $-0.38 \pm 2.26 \%$ & 0.29 & 92.00 \\
\hline & B51 & Trained $=11 ;$ Untrained $=11$ & $V_{\mathrm{m}}$ & $-2.44 \pm 3.78 \%$ & $0.85 \pm 1.84 \%$ & 0.69 & 54.00 \\
\hline & & Trained $=11 ;$ Untrained $=9$ & $R_{\mathrm{m}}$ & $3.59 \pm 2.32 \%$ & $16.7 \pm 6.08 \%$ & 0.11 & 28.00 \\
\hline \multirow[t]{3}{*}{ 5-HT application } & TSN & $5-\mathrm{HT}=8 ; \mathrm{ASW}=7$ & $V_{\mathrm{m}}$ & $-5.64 \pm 1.32 \%$ & $-3.87 \pm 3.99 \%$ & 0.19 & 16.50 \\
\hline & B51 & $5-\mathrm{HT}=9 ; \mathrm{ASW}=6$ & $V_{\mathrm{m}}$ & $2.13 \pm 2.40 \%$ & $2.21 \pm 2.82 \%$ & 0.82 & 16.00 \\
\hline & & $5-\mathrm{HT}=8 ; \mathrm{ASW}=6$ & $R_{\mathrm{m}}$ & $-16.20 \pm 4.50 \%$ & $6.63 \pm 3.86 \%$ & $<0.05$ & 4.00 \\
\hline
\end{tabular}

Values are expressed as mean \pm SEM. The Mann-Whitney $U$-test was used to compare changes between groups. Statistical significance was set at $P<0.05$.

effect of 5-HT on $V_{\mathrm{m}}$ in either TSNs, or B51 $15 \mathrm{~min}$ after treatment (Table 1). We observed a significant decrease of $R_{\mathrm{m}}$ in B51 at $15 \mathrm{~min}$ after 5-HT application (Table 1). However, there was no significant correlation between the change in $R_{\mathrm{m}}$ and the change in burst threshold in B51 treated with 5-HT $(r=-0.18, P=0.62$, Spearman Rank Order Correlation). Finally, we analyzed the effect of 5-HT on the inhibitory synaptic input to B51. The average IPSP amplitude did not change after 5 -HT bath application (pretest: $-6.06 \pm$ $1.10 \mathrm{mV}$; post-test: $-4.72 \pm 0.60 \mathrm{mV}, n=8 ; P=0.20 ; W=20.00$; Wilcoxon signed rank test).

\section{Discussion}

This study illustrates the successful development of the first in vitro analog that expresses learning-induced cellular correlates in multiple behaviorally relevant neural circuits. In particular, increased TSN excitability in the TSWR circuit, and decreased B51 excitability in the feeding circuit were observed following in vitro training consisting of P8/P9 electrical stimulation.

The configuration of this in vitro analog (Fig. 1A) was obtained by retaining the pleural-pedal ganglia (site of the TSWR circuit and origin of the nerves P8 and P9; Cleary et al. 1995), the buccal ganglion (site of the feeding circuit; Cropper et al. 2004) and the cerebral ganglion that connects them and contains interneurons activated by sensitizing stimuli (Marinesco et al. 2004) as well as command-like interneurons that control the feeding circuit (Cropper et al. 2004). This preparation was derived from a previous in vitro analog of sensitization, consisting exclusively of the pleural-pedal ganglia, which expresses correlates of sensitization (e.g., increased TSN excitability and synaptic facilitation of the sensorimotor neuron synapse) following P8/P9 electrical stimulation (Goldsmith and Byrne 1989; Zhang et al. 1994; Liao et al. 1999).

Learning-dependent changes in excitability were originally identified in TSNs and B51 using a four-trial in vivo sensitization protocol that induces long-term behavioral modifications lasting at least $24 \mathrm{~h}$ (Cleary et al. 1998; Shields-Johnson et al. 2013). For this study, we used an in vitro protocol, which closely matched the in vivo sensitization training in number of trials, stimulus intensity, and temporal pattern of nerve stimulation (Fig. 1B2; see Materials and Methods for details). Previous use of this in vitro protocol in the isolated pleural-pedal ganglia revealed cellular correlates of sensitization, including reduction of the net outward current in TSNs (Noel et al. 1991) and synaptic facilitation of the synapse between TSNs and tail motor neurons (Goldsmith and Byrne 1989; Zhang et al. 1994). Our findings indicate that another long-term correlate of sensitization is expressed following in vitro training: increase of TSN excitability (Fig. 4A; Cleary et al. 1998). The increase of TSN excitability was not accompanied by modifica- tions in resting membrane potential, which is consistent with previous results obtained with both in vivo (Cleary et al. 1998) and in vitro sensitization protocols (Zhang et al. 1994), and with 5-HT treatments used in lieu of training (Dale et al. 1987; Baxter and Byrne 1990; Liu et al. 2011).

In this study, we have also demonstrated that the in vitro training induced modifications outside the TSWR neural circuit, precisely a decreased excitability in B51, an element of the feeding neural circuit (Fig. 4B). The decreased excitability in B51 was not accompanied by modifications of resting membrane potential, input resistance or synaptic input, thus recapitulating the results
A1

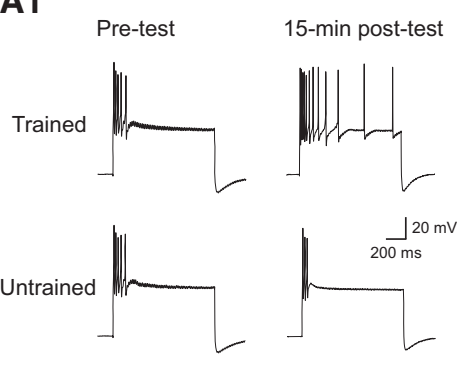

B1
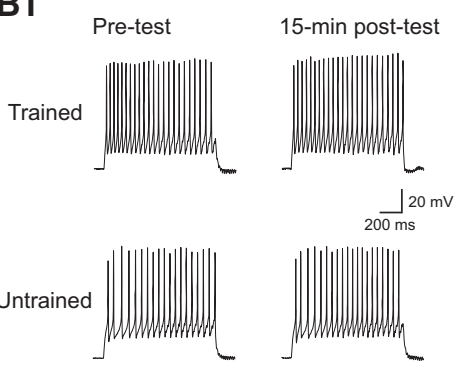

A2

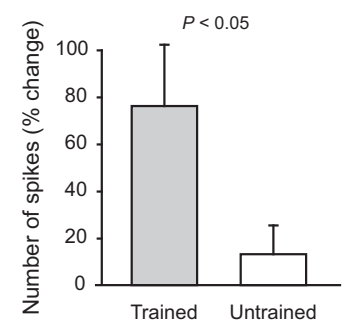

B2

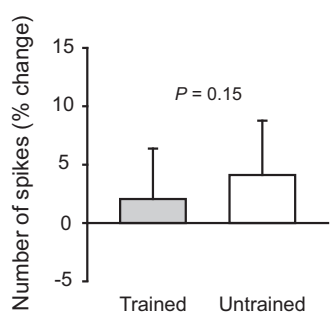

Figure 3. Single-trial in vitro training increased TSN excitability, but did not alter the excitability of neuron B4/5. Using the short-term in vitro analog illustrated in Figure $1 \mathrm{~A}$ and $\mathrm{B} 1$, the effects of in vitro training were examined on B4/5. The excitability of TSN and B4/5 was assessed prior to and 15 min after treatment. (A1) Sample traces of TSN firing from trained and untrained preparations. (A2) Summary data illustrate that the single-trial in vitro training significantly increased TSN excitability (change in number of spikes, trained: $76.42 \pm 26.17 \%$ of pretest; $n=28$; untrained: $13.20 \pm 12.25 \%$ of pretest; $n=29 ; \quad P<0.05 ; \quad U=282.50$; Mann-Whitney $U$-test). (B1) Sample traces of B4/5 firing from trained and untrained preparations. (B2) Summary data illustrate that B4/5 excitability did not differ between trained and untrained preparations (change in number of spikes, trained: $2.06 \pm 4.32 \%$ of pretest; $n=34$; untrained: $4.12 \pm 4.66 \%$ of pretest; $n=35 ; P=0.15 ; \quad U=474.00$; Mann-Whitney U-test). 
A1

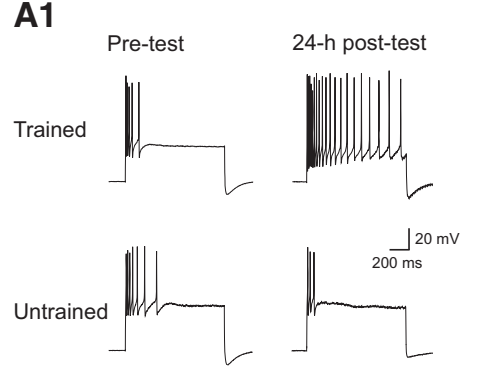

B1
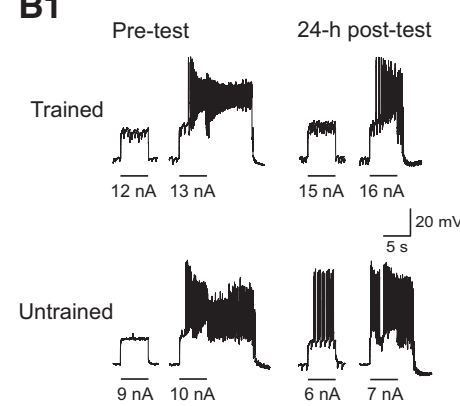

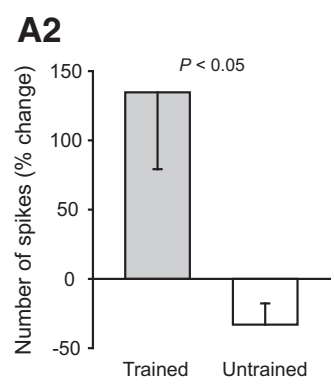

B2

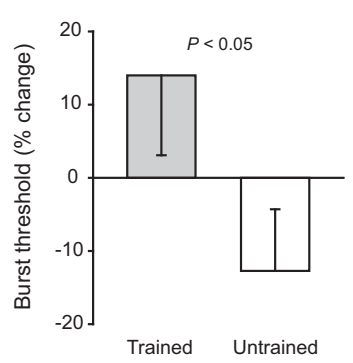

Figure 4. Four-trial in vitro training induced a concurrent increase of TSN excitability and a decrease of B51 excitability $24 \mathrm{~h}$ after training. (A1) Sample traces of TSN firing from trained and untrained preparations. (A2) Summary data illustrate that the four-trial in vitro training significantly increased TSN excitability (change in number of spikes, trained: $134.64 \pm$ $55.47 \%$ of pretest; $n=14$; untrained: $-33.07 \pm 15.30 \%$ of pretest; $n=17$; $P<0.05 ; U=60.00$; Mann-Whitney $U$-test). (B1) Sample traces of B51 burst threshold from trained and untrained preparations. (B2) Summary data illustrate that the four-trial in vitro training significantly increased B51 burst threshold (change in burst threshold, trained: $13.99 \pm 10.92 \%$ of pretest; $n=11$; untrained: $-12.72 \pm 8.42 \%$ of pretest; $n=11 ; P<$ 0.05; $U=29.50$; Mann-Whitney $U$-test).

obtained with a four-trial in vivo sensitization protocol (ShieldsJohnson et al. 2013; Hernandez et al. 2017). Several lines of evidence suggest that decreased B51 excitability is intrinsic: (1) the lack of training-induced changes in resting membrane potential and input resistance rules out the possibility that modulation of either tonic synaptic inputs to B51 or electrical coupling between B51 and other cells might contribute to the observed decreased excitability. (2) The lack of training-induced changes in IPSP amplitude (Shields-Johnson et al. 2013; this study) excludes the contribution of synaptic plasticity to decreased B51 excitability. (3) The plateau potential in B51 is known to be an intrinsic feature that is retained in isolated cultured cells (Brembs et al. 2002; Mozzachiodi et al. 2008). (4) Recent findings revealed that B51 decreased excitability induced by in vivo training is sustained by a training-induced nonsynaptic modulation of voltage-gated $\mathrm{Na}^{+}$ channels (Hernandez et al. 2017).

From the four-trial in vitro protocol, we derived a single-trial in vitro training (Fig. 1B1), which is analogous to an in vivo protocol that induces short-term sensitization and feeding suppression (Acheampong et al. 2012). This single-trial in vitro training induced concurrent increased TSN excitability (Fig. 2A) and decreased B51 excitability (Fig. 2B), with no effect on the resting properties of either neurons. Like the long-term modifications described above, these excitability changes are consistent with the coexpression of short-term sensitization and feeding suppression, respectively (Acheampong et al. 2012). Overall, the in vitro analog developed in this study coexpressed excitability changes in TSNs and B51 in two distinct temporal domains: short term (i.e., 15 min after the single-trial training; Fig. 2), and long term (24 h after

the four-trial training; Fig. 4), indicating that the mechanisms of memory storage (i.e., plasticity of intrinsic excitability) are conserved from the short term to the long term.

To expand the application of the in vitro analog, we began to examine to which extent the effects of sensitization training are distributed within the feeding neural circuit. We investigated the role of neurons $\mathrm{B} 4 / 5$, which are involved in motor program switching (Warman and Chiel 1995, Jing and Weiss 2001). Previous results revealed that the membrane properties of $\mathrm{B} 4 / 5$ were not altered following in vivo or in vitro paradigms of appetitive classical conditioning (Lechner et al. 2000; Mozzachiodi et al. 2003). A similar outcome was observed in this study: although in vitro training induced the expected increase of TSN excitability (Fig. 3A), it did not induce changes in B4/5 membrane properties (Fig. 3B). This result indicates that B4/5 is not a locus of plasticity underlying the feeding suppression produced by sensitization training. By providing a "negative control" of neuronal plasticity, these findings suggest that the changes induced by sensitization training are not generalized throughout the feeding neural circuit, but they are restricted to specific neurons, such as B51. It is important to note that B51 is unlikely the sole site of plasticity induced by sensitizing stimuli within the feeding neural circuit. Our developed in vitro analog will allow us to further explore additional sites of plasticity underlying the learning-induced feeding suppression, including pattern-initiating neurons in the buccal ganglion, such as B63 and B31/32
A1

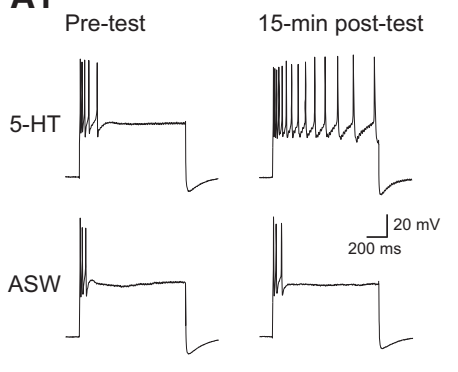

B1

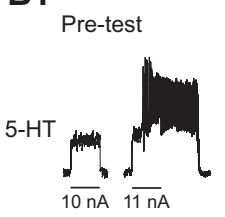

ASW 15-min post-test

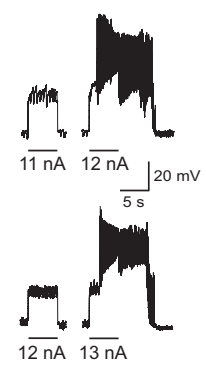

A2

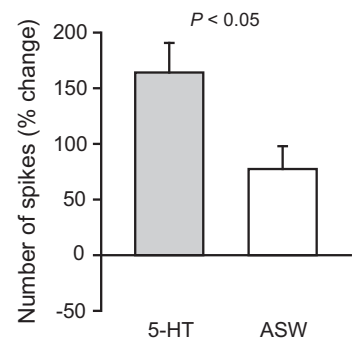

B2

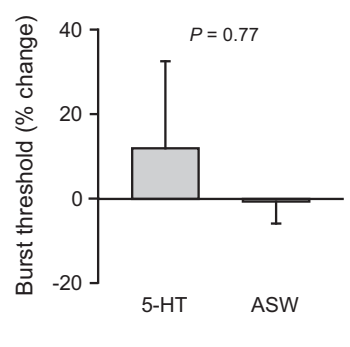

Figure 5. 5-HT differentially modulates excitability of TSNs and B51. The in vitro configuration and the testing protocol were identical to the ones illustrated in Figure $1 \mathrm{~A}$ and $\mathrm{B} 1$ with the exception that treatment with $50 \mu \mathrm{M} 5-\mathrm{HT}$ replaced P8/P9 electrical stimulation. Excitability of TSN and B51 was assessed prior to and 10 min after a 5-min treatment with either 5-HT or ASW. (A1) Sample traces of TSN firing from preparations treated with 5-HT or ASW. (A2) Summary data illustrate that 5-HT significantly increased TSN excitability change in number of spikes, 5-HT: $164.14 \pm 26.55 \%$ of pretest; $n=8$; ASW: $77.36 \pm 20.55 \%$ of pretest; $n=$ 7; $P<0.05 ; U=9.00$; Mann-Whitney $U$-test). (B1) Sample traces of B51 burst threshold from preparations treated with 5-HT or ASW. (B2) Summary data illustrate that B51 burst threshold did not differ between preparations treated with either 5-HT or ASW (change in burst threshold, 5 -HT: $11.95 \pm 20.53 \%$ of pretest; $n=9$; ASW: $-0.63 \pm 5.15 \%$ of pretest; $n$ $=6 ; P=0.77 ; U=24.00 ;$ Mann-Whitney U-test). 
(Nargeot and Simmers 2012), as well as cerebral command-like interneurons (CBIs), which are capable of driving the feeding neural circuit (Cropper et al. 2004).

Previous work in Aplysia has established that 5-HT mediates sensitization of withdrawal reflexes and its underlying cellular correlates (Brunelli et al. 1976; Glanzman et al. 1989; Levenson et al. 2000; Marinesco and Carew 2002; Byrne and Hawkins 2015). Indeed, 5-HT can serve as analog of sensitization training in inducing facilitation of the sensory-to-motor neuron synapses and increased sensory neuron excitability (Walters et al. 1983a,b; Dale et al. 1987; Baxter and Byrne 1990; Byrne and Kandel 1996; Liu et al. 2011, Byrne and Hawkins 2015). However, recent results indicate that 5 -HT does not mimic sensitization training in inducing feeding suppression or decreased B51 excitability (Shields-Johnson et al. 2013).

The in vitro analog developed in this study recapitulates the differential effects that 5-HT exerts on sensitization and feeding suppression: 5-HT treatment induced an increase of TSN excitability (Fig. 5A), but did not modify B51 excitability (Fig. 5B), or the inhibitory synaptic input to the cell. 5-HT bath application induced an unexpected significant decrease of $R_{\mathrm{m}}$ in B51, but this change did not correlate with the change in burst threshold and was not sufficient to significantly alter B51 excitability. Current evidence indicates that $R_{\mathrm{m}}$ is not altered in $\mathrm{B} 51$ by either in vivo (Shields-Johnson et al. 2013) or in vitro sensitizing stimuli (this study), which are known to trigger 5-HT release from interneurons in the pleural-pedal and cerebral ganglia (Marinesco and Carew 2002; Marinesco et al. 2004; Philips et al. 2011). An explanation for the discrepancy between the effects of sensitization training and 5-HT bath application may reside in the different modes of 5-HT action: in vivo and in vitro sensitizing stimuli lead to the local release of 5-HT in the neuropil (Marinesco and Carew 2002), whereas 5-HT bath application may have more diffused effects within the feeding neural circuit. Indeed, previous findings indicate that 5-HT bath application alters the properties of multiple feeding neurons, such as decreasing the input resistance of neuron B31/32 and the excitability of neuron B4/5 (Kabotyanski et al. 2000). In addition, we cannot rule out the possibility that the decrease of B51 $R_{\mathrm{m}}$ may reflect a modulation of the electrical coupling between B51 and other buccal neurons (Plummer and Kirk 1990; Mozzachiodi et al. 2013; Sasaki et al. 2013) induced by 5-HT bath application.

The different effects of 5-HT on the excitability of TSNs and B51 strengthen the view that distinct biochemical pathways are responsible for the changes occurring in the neural circuits controlling the TSWR and feeding, following sensitizing stimuli. Despite the extensive knowledge that 5-HT release modulates the activity of the withdrawal circuits (Marinesco and Carew 2002; Marinesco et al. 2004; Byrne and Hawkins 2015), the training-induced pathway that leads to the decrease of B51 excitability remains uncharacterized and future investigations are necessary to identify the underlying modulator(s). A putative candidate is nitric oxide (NO), which in Aplysia mediates forms of nociceptive sensitization (Lewin and Walters 1999) and aversive classical conditioning (Antonov et al. 2007), and modulates feeding and its neural circuit (Susswein and Chiel 2012). Current findings indicate that NO contributes to the training-induced suppression of feeding. Systemic in vivo injection of the NO synthase inhibitor L-NAME prevented the expression of feeding suppression following either single-trial or four-trial sensitization protocols (Farruggella et al. 2015). Recent results also indicate that, at the cellular level, iontophoretic injections of cGMP decreases B51 excitability in a manner analogous to that produced by sensitization training, with one injection reducing excitability for at least $15 \mathrm{~min}$, and four injections, spaced $30 \mathrm{~min}$ apart, reducing excitability for at least $24 \mathrm{~h}$ (Goldner et al. 2016). These findings suggest that a NO-cGMP pathway may mediate the training-induced feeding suppression and the underlying decreased of B51 excitability. Future experiments will use the in vitro analog to characterize the biochemical steps linking sensitizing stimuli to decreased B51 excitability.

The use of in vitro analogs derived from vertebrate and invertebrate preparations has greatly contributed to revealing the occurrence of distributed forms of learning-dependent neuronal plasticity in identified neural circuits. For example, in the mammalian cerebellum, long-term potentiation (LTP) and long-term depression (LTD) can be induced by different learning rules in distinct cerebellar regions (Suvrathan et al. 2016). Similarly, in the leech Hirudo, multiple forms of LTP and LTD (Burrell and Sahley 2004) and bidirectional changes in excitability (Burrell et al. 2001) are induced in defensive neural circuits by patterns of electrical stimulation in vitro. In Aplysia, bidirectional modifications of synaptic efficacy and neuronal excitability have been identified at the level of sensory neurons and interneurons responsible for defensive withdrawal reflexes of the tail, siphon and gills, following in vitro analogs of sensitization training (Trudeau and Castellucci 1993, Cleary et al., 1995; Cohen et al. 1997). Equivalent findings have been reported in the neural circuit controlling feeding in Aplysia in which in vitro analogs of appetitive classical and operant conditioning both express forms of synaptic and intrinsic plasticity occurring at multiple sites (for review, see Nargeot and Simmers 2012; Mozzachiodi et al. 2013). However, in the above in vitro analogs, the learning-induced plasticity is expressed within individual circuits or in multiple circuits with similar functions, such as those controlling defensive withdrawals of tail, siphon, and gills in Aplysia (Cleary et al. 1995).

The novelty of the in vitro analog described in this study is that it expresses distributed cellular changes occurring concomitantly in multiple neural circuits of very different nature: defensive (TSWR) and appetitive (feeding), following exposure to aversive stimuli. In particular, in vitro sensitization training induces opposite changes in excitability in key neurons within the TSWR and feeding neural circuits, which are analogous to those measured following in vivo training and are consistent with the observed learning-dependent behavioral modifications.

In conclusion, this in vitro analog, expressing cellular correlates of both sensitization and feeding suppression, offers an unprecedented opportunity to compare and contrast learning-induced changes occurring simultaneously in multiple neural circuits. This goal will be achieved through the use of procedures and techniques that are not feasible in vivo, such as local/global activation or inhibition of individual biochemical pathways, as well as cytosolic/nuclear manipulations of individual neurons in different neural circuits. This approach will be instrumental in exploring important aspects of learning-induced neuronal plasticity, including: (1) the relationship between the cellular and molecular underpinnings underlying modifications occurring in different neural circuits, (2) the comparison between short-term and long-term mechanisms, and (3) the comparison between changes induced by learning paradigms using stimuli of different modalities. The latter aspect is particularly relevant for B51, the excitability of which is modulated in opposite directions by appetitive (Nargeot et al. 1999a,b; Brembs et al. 2002; Mozzachiodi et al. 2008) and aversive stimuli (Shields-Johnson et al. 2013; this study).

\section{Materials and Methods}

\section{General methods}

Wild-caught Aplysia californica (130-180 g) were obtained from Marinus Scientific and South Coast Bio-Marine, and housed individually in perforated plastic cages in two aquaria (Acquatic Enterprises Inc.) of continuously circulating $15^{\circ} \mathrm{C}$ aquarium seawater (Instant Ocean) on a 12-h light-dark cycle. A period of 
acclimation of at least $3 \mathrm{~d}$ upon arrival was allowed before animals were utilized, to minimize the effects of stress caused by shipping and handling (Levenson et al. 1999). Animals were fed $\sim 1 \mathrm{~g}$ of dried seaweed (Emerald Cove Organic Pacific Nori) three times a week. Animals were food deprived for $2-3 \mathrm{~d}$ prior to the experiment.

\section{Dissection}

Prior to each experiment, the general health and motivational state of the animal was tested by presenting it with a food stimulus. The animal was included in the study only if it generated bites in response to the food stimulus. Aplysia were anesthetized by injecting a volume of isotonic $\mathrm{MgCl}_{2}$ (equivalent to $50 \%$ of the weight of the animal) through the foot. The animal was placed ventral side up in a dissecting tray and a longitudinal incision was made, along the midline of the foot to expose the central nervous system. The incision extended approximately one-third the length of the animal to the margin of the lips. The buccal, cerebral, and pleural-pedal ganglia were removed together with their interganglionic connectives to retain the critical components of the neural circuits controlling the TSWR and feeding (Fig. 1A; Cleary et al. 1995; Cropper et al. 2004). A few centimeters of the left or right pedal nerves P8 and P9, which innervate the lateral and posterior body wall and tail (Fig. 1A; Hening et al. 1979; Walters et al. 2004), were retained for extracellular nerve stimulation. The side of nerves P8 and P9 origin (left or right) was randomly selected for each preparation. One of the two buccal nerves 2,3 (denoted n.2,3) was retained for identification of neuron B51 (Nargeot et al. 1999a; Shields-Johnson et al. 2013). All other nerves were cut short.

The isolated ganglia were transferred to a Sylgard-coated petri dish, containing high-divalent artificial seawater (ASW) composed of $210 \mathrm{mM} \mathrm{NaCl}, 10 \mathrm{mM} \mathrm{KCl}, 145 \mathrm{mM} \mathrm{MgCl}_{2}, 20 \mathrm{mM} \mathrm{MgSO}_{4}, 33$ $\mathrm{mM} \mathrm{CaCl}_{2}$, and $10 \mathrm{mM}$ HEPES, with the $\mathrm{pH}$ adjusted to 7.5 using $\mathrm{NaOH}$ (Mozzachiodi et al. 2003). The cerebral and the pleuralpedal ganglia were pinned ventral-side up. In this configuration, the buccal ganglion was flipped to expose the rostral side by bringing the radula nerve up over the ganglion and pinning it between the esophageal nerves. The sheath on the ventral surface of the pleural ganglion on the side of the retained nerves P8 and P9 was surgically removed using fine forceps and scissors to expose the somata of TSNs in the VC cluster (Fig. 1A; Zhang et al. 1994; Cleary et al. 1998). The sheath was also removed from the rostral surface of both buccal hemiganglia to expose the somata of B51 and B4/5 (Nargeot et al. 1999a; Mozzachiodi et al. 2003). Individual bipolar extracellular stimulating electrodes were placed along P8, P9, and n.2,3 and were isolated from the bath with Vaseline (Nargeot et al. 1997; Mozzachiodi et al. 2003). The highdivalent ASW was replaced by normal ASW (composed of $450 \mathrm{mM}$ $\mathrm{NaCl}, 10 \mathrm{mM} \mathrm{KCl}, 30 \mathrm{mM} \mathrm{MgCl}_{2}, 20 \mathrm{mM} \mathrm{MgSO}_{4}, 10 \mathrm{mM} \mathrm{CaCl}_{2}$, and $10 \mathrm{mM}$ HEPES, with $\mathrm{pH}$ adjusted to 7.5 using $\mathrm{NaOH}$; Mozzachiodi et al. 2003) and the preparation was rested for 30 min. The recording chamber was maintained at $15^{\circ} \mathrm{C}$ throughout the electrophysiological recordings and experimental treatments with a feedback-controlled cooling device (Model BTC-100/ BTC-S, Bioscience Tools, CA).

\section{Intracellular recordings}

All intracellular recordings were conducted in current-clamp mode using a preamplifier (IX2-700, Dagan Corporation). Fine-tipped glass microelectrodes were filled with $3 \mathrm{M}$ potassium acetate (resistance $10-12 \mathrm{M} \Omega$ ).

\section{Neuron B51}

The soma of neuron B51 was identified based on its relative size and position within the buccal ganglion and the occurrence of its characteristic intrinsically generated all-or-nothing bursts of action potentials, known as plateau potentials (Plummer and Kirk 1990; Nargeot et al. 1999a). If B51 did not fire spontaneously or in response to brief intracellular depolarizing pulses, then n.2,3 was briefly stimulated (1-2 sec of $10 \mathrm{~V}, 0.5 \mathrm{msec}$ pulses at $4 \mathrm{~Hz}$ ), and the cell's response was used to aid in identification. Two-electrode current-clamp was used for intracellular recordings from B51. The $V_{\mathrm{m}}$ was recorded $5 \mathrm{~min}$ after impalement. Cells were included in the study only if they displayed a $V_{\mathrm{m}}$ of at least $-45 \mathrm{mV}$ (Shields-Johnson et al. 2013). B51 was then current clamped at $-60 \mathrm{mV}$, and the input resistance $\left(R_{\mathrm{m}}\right)$ and the excitability was measured. $R_{\mathrm{m}}$ was calculated from the voltage drop elicited by a 5-sec, 5-nA hyperpolarizing current pulse (Nargeot et al. 1999a). The excitability of B51 was assessed by measuring the neuron's burst threshold, defined as the minimum amount of depolarizing current necessary to elicit a plateau potential outlasting the duration of the current pulse (Nargeot et al. 1999a). B51 burst threshold was determined by delivering a series of 5-sec depolarizing current pulses, beginning at 5-nA intensity and increasing in intensity in 1-nA increments, at 10-sec intervals (Nargeot et al. 1999a,b). Cells that did not exhibit a plateau potential by $25 \mathrm{nA}$ were excluded from the study. Inhibitory synaptic potentials (IPSPs), originating from presynaptic neurons within the feeing neural circuit, including B52 (Plummer and Kirk 1990; Nargeot et al. 2002), were observed in B51 in about $77 \%$ of the preparations used in this study. The contribution of this inhibitory synaptic input to the training-induced decrease of B51 excitability was assessed by analyzing the magnitude of the IPSPs in the preparations that were trained with either the single-trial or the four-trial in vitro training, as well in the preparations that were treated with 5-HT. We measured the amplitude of the IPSPs occurring during the 5-sec depolarizing current pulses, using the current intensity immediately prior to the burst threshold. The IPSP amplitude was averaged before and after training/5-HT treatment.

\section{TSNs}

One-electrode current-clamp was used for intracellular recordings from TSNs (Zhang et al. 1994; Cleary et al. 1998; Chin et al. 1999; Liu et al. 2011). We opted for the use of a single electrode for recording and stimulation because of the risk of dislodging electrodes during the delivery of the in vitro training or 5-HT bath application and following washout. The somata of TSNs were identified based on their position in the VC cluster of the pleural ganglion, and the occurrence of action potentials in response to a single 3-msec stimulus delivered to nerve P9 (Cleary et al. 1998; Walters et al. 2004). $V_{\mathrm{m}}$ was recorded $5 \mathrm{~min}$ after impalement. Cells were included in the study only if they displayed a resting membrane potential of at least $-35 \mathrm{mV}$ (Zhang et al. 1994). TSNs were subsequently clamped at $-45 \mathrm{mV}$ (Baxter and Byrne 1990; Chin et al. 1999) and their excitability was assessed as the number of spikes evoked by a 1-sec, 2-nA depolarizing current pulse (Cleary et al. 1998).

\section{Neuron $B 4 / 5$}

The soma of B4/5 was identified in the buccal ganglion based on size, location and the occurrence of spontaneous excitatory synaptic inputs (Gardner 1977; Church and Lloyd 1994). B4/5 membrane properties were measured using two-electrode current clamp. $V_{\mathrm{m}}$ was recorded $5 \mathrm{~min}$ after impalement. Cells were included in the study only if they displayed a resting membrane potential of at least $-45 \mathrm{mV}$. B $4 / 5$ was subsequently clamped at $-55 \mathrm{mV}$ for the assessment of $R_{\mathrm{m}}$ and excitability. $R_{\mathrm{m}}$ was calculated from the voltage drop induced by injecting 5-sec, 5-nA pulse of hyperpolarizing current. Excitability was measured as the number of action potentials elicited by a 1-sec, 10-nA depolarizing current pulse.

\section{Experimental protocols}

\section{Single-trial in vitro sensitization training}

In Aplysia, the effects of sensitization training on TSWR and TSN excitability are restricted to the trained side (Scholz and Byrne 1987; Zhang et al. 1994; Cleary et al., 1998). Therefore, in this study, only TSNs ipsilateral to the retained nerves P8 and P9 were used (Fig. 1A; Zhang et al. 1994). However, because feeding suppression and the subsequent decreased excitability in B51 are not 
lateralized (Acheampong et al. 2012; Shields Johnson et al. 2013), $\mathrm{B} 51$ and B4/5 were used regardless of whether they were ipsilateral or contralateral to the retained nerves P8 and P9 (Fig. 1A). Membrane properties $\left(V_{\mathrm{m}}, R_{\mathrm{m}}\right.$ and burst threshold for B51; $V_{\mathrm{m}}$, $R_{\mathrm{m}}$ and number of action potentials for $\mathrm{B} 4 / 5 ; V_{\mathrm{m}}$ and number of action potentials for TSNs) were initially measured (pretest; Fig. 1B1). The current clamp was then removed and the ganglia were then rested for 10 min before delivery of the single-trial training protocol. The single-trial training consisted of $10-\mathrm{sec}, 1 \mathrm{~Hz}$, train of ten $500-\mathrm{msec}, 60-\mathrm{Hz}, 60-\mathrm{V}$ impulses delivered simultaneously to the retained nerves P8 and P9 (Zhang et al. 1994). Controls consisted of untrained preparations that did not receive any electrical stimulation to P8/P9. Preparations were randomly assigned to receive training or serve as untrained controls. Cells were held throughout training. Fifteen minutes after training, membrane properties were measured again (post-test; Fig. 1B1) to assess changes induced by training.

\section{Four-trial in vitro sensitization training}

TSNs and B51 were selected following the same procedure used for the single-trial training. Membrane properties of B51 and TSNs were initially measured as described above (pretest; Fig. 1B2), and then the electrodes were removed. Prior to removing the electrode from the recorded TSN, the cell location was marked by iontophoretically injecting $4-5$ cells immediately surrounding it with Fast Green dye (10-nA hyperpolarizing current for $\sim 30 \mathrm{sec}$, or until the soma was dark green in color; Scholz and Byrne 1987; Zhang et al. 1994; Sherff and Carew 2004). Images of pleural and buccal ganglia were also taken (MD800E microscope-mounted USB camera, AmScope), and the cells were annotated in Microsoft Paint to aid in the identification of B51 and TSN for 24-h post-test measurements of membrane properties (Fig. 1B2). Once TSNs were labeled and images were taken (10-15 min), ASW was exchanged with modified L-15 culture medium consisting of L-15 medium (Sigma-Aldrich) supplemented with (in $\mathrm{mM}$ ) $\mathrm{NaCl} 293.1, \mathrm{KCl}$ 4.6, $\mathrm{MgCl}_{2} 26, \mathrm{MgSO}_{4}$ 18.9, $\mathrm{CaCl}_{2} 10.9$, HEPES 30 and $0.10 \mathrm{~g} / \mathrm{L}$ of streptomycin (Sigma-Aldrich) and $0.12 \mathrm{~g} / \mathrm{L}$ of penicillin-G (Sigma-Aldrich), $\mathrm{pH}$ adjusted to 7.5 with $\mathrm{NaOH}$ (Mozzachiodi et al. 2008). To monitor the efficacy of subsequent training, one of the large neurons in the pleural ganglion adjacent to the medial edge of the $\mathrm{VC}$ cluster (that reliably responded to $\mathrm{p} 8 / \mathrm{p} 9$ stimulation with fluctuations in the $V_{\mathrm{m}}$; Liao et al. 1999) was impaled and held throughout the training period. The in vitro training (or no training) protocol began $10 \mathrm{~min}$ after the ASW exchange. The four-trial training consisted of four 10-sec trains of P8/P9 electrical stimulation (each identical to that used for the single-trial training) spaced 30 min apart (Goldsmith and Byrne 1989; Zhang et al. 1994). Controls consisted of untrained preparations that did not receive any electrical stimulation to P8/P9. Preparations were randomly assigned to either receive training or serve as untrained controls. Once the training was concluded, the extracellular stimulating electrodes were gently removed and the recording chamber was covered and stored at $15^{\circ} \mathrm{C}$ for 23.5 h. On the next day, ganglia were rinsed in normal ASW and allowed to rest for $30 \mathrm{~min}$. Post-test measurement of membrane properties commenced $24 \mathrm{~h}$ after training (Fig. 1B2).

\section{Effects of 5-HT application on the membrane properties of B51 and TSNs}

In the last experiment of this study, we examined whether 5-HT application could successfully replace the single-trial training in inducing concomitant excitability changes in B51 and TSNs. Because previous findings indicate that 5-HT increases the excitability of TSNs (Walters et al. 1983b), we used increased excitability as a "positive control" for the effectiveness of 5-HT treatment. Serotonin hydrochloride (Alfa Aesar) was dissolved in ASW to make a 50-mM stock. Stock solutions were made fresh daily and stored at $4^{\circ} \mathrm{C}$. The stock solution was diluted to $50 \mu \mathrm{M}$ in ASW prior to being bath applied to the ganglia. The experimental design was identical to the one used for the in vitro single-trial training with the exception that 5-HT treatment replaced the training. Membrane properties of B51 and TSNs were measured as described above (pretest). Five minutes after the measurements were completed, preparations were randomly assigned to receive treatment with either 5-HT or vehicle (ASW). For the 5-HT treated preparations, $50 \mathrm{~mL}$ of $50 \mu \mathrm{M} 5$-HT was manually exchanged at a rate of $\sim 10 \mathrm{~mL} / \mathrm{min}$ by slowly adding the 5 -HT solution with a transfer pipette to one side of the petri dish while simultaneously removing an equal volume from the opposite side of the petri dish. The application of 5-HT took $5 \mathrm{~min}$. At the end of this period, 5-HT was washed out of with $50 \mathrm{~mL}$ of ASW in an identical manner. Controls were handled identically, except that ASW was used in place of 5-HT. Ten minutes after the end of the washout (i.e., 15 min after the beginning of treatment with 5-HT or ASW), membrane properties were measured again (post-test) to assess changes induced by treatment.

\section{Statistical analysis}

For each membrane property of each neuron recorded, the percent change was calculated as [(post - pre/pre $) \times 100]$ to assess modifications due to training and/or treatment. We were not able to collect every measurement from B51, B4/5 and TSNs in each preparation. This discrepancy was caused primarily by: (1) the inability to find B51, B4/5 and TSNs in every preparation, (2) the inability to maintain recordings until the end of the experiment. The MannWhitney $U$-test was used to compare changes in membrane properties between trained/treated and untrained/control groups (Mozzachiodi et al. 2003; Lorenzetti et al. 2006). The Wilcoxon signed rank test was used to analyze the effect of in vitro training protocols and 5-HT bath application on the amplitude of B51 IPSP. Finally, the Spearman rank correlation coefficient $r$ was used to analyze the correlation between changes in $R_{\mathrm{m}}$ and changes in burst threshold in preparations treated with 5-HT. Statistical significance was set at $P<0.05$. Data analyses were performed using the statistical package of SigmaPlot 11.0 (Jandel Scientific).

\section{Acknowledgments}

This work was supported by NSF grant IOS-1120304 (R.M.) and NIH-NIGMS grant SC3GM111188 (R.M.).

\section{References}

Acheampong A, Kelly K, Shields-Johnson M, Hajovsky J, Wainwright M, Mozzachiodi R. 2012. Rapid and persistent suppression of feeding behavior induced by sensitization training in Aplysia. Learn Mem 19: 159-163.

Antonov I, Kandel ER, Hawkins RD. 1999. The contribution of facilitation of monosynaptic PSPs to dishabituation and sensitization of the Aplysia siphon withdrawal reflex. J Neurosci 19: 10438-10450.

Antonov I, Antonova I, Kandel ER, Hawkins RD. 2001. The contribution of activity-dependent synaptic plasticity to classical conditioning in Aplysia. J Neurosci 21: 6413-6422.

Antonov I, Antonova I, Kandel ER, Hawkins RD. 2003. Activity-dependent presynaptic facilitation and Hebbian LTP are both required and interact during classical conditioning in Aplysia. Neuron 37: 135-147.

Antonov I, Ha T, Antonova I, Moroz LL, Hawkins RD. 2007. Role of nitric oxide in classical conditioning of siphon withdrawal in Aplysia. J Neurosci 27: 10993-11002.

Baxter DA, Byrne JH. 1990. Differential effects of cAMP and serotonin on membrane current, action-potential duration, and excitability in somata of pleural sensory neurons of Aplysia. J Neurophysiol 64: 978-990.

Brembs B, Lorenzetti FD, Reyes FD, Baxter DA, Byrne JH. 2002. Operant reward learning in Aplysia: neuronal correlates and mechanisms. Science 296: $1706-1709$.

Brunelli M, Castellucci V, Kandel ER. 1976. Synaptic facilitation and behavioral sensitization in Aplysia: possible role of serotonin and cyclic AMP. Science 194: 1178-1181.

Burrell BD, Sahley CL. 2004. Multiple forms of long-term potentiation and long-term depression converge on a single interneuron in the leech CNS. J Neurosci 24: 4011-4019.

Burrell BD, Sahley CL, Muller KJ. 2001. Non-associative learning and serotonin induce similar bi-directional changes in excitability of a neuron critical for learning in the medicinal leech. J Neurosci 21: 1401-1412.

Byrne JH. 1987. Cellular analysis of associative learning. Physiol Rev 67: 329-439. 
Byrne JH, Hawkins RD. 2015. Nonassociative learning in invertebrates. Cold Spring Harb Perspect Biol 7: a021675.

Byrne JH, Kandel ER. 1996. Presynaptic facilitation revisited: state and time dependence. J Neurosci 16: 425-435.

Chin J, Angers A, Cleary LJ, Eskin A, Byrne JH. 1999. TGF-b1 in Aplysia: role in long-term changes in the excitability of sensory neurons and distribution of TbR-II-like immunoreactivity. Learn Mem 6: 317-330.

Church PJ, Lloyd PE. 1994. Activity of multiple identified motor neurons recorded intracellularly during evoked feeding-like motor programs in Aplysia. J Neurophysiol 72: 1794-1809.

Cleary LJ, Byrne JH, Frost WN. 1995. Role of interneurons in defensive withdrawal reflexes in Aplysia. Learn Mem 2: 133-151.

Cleary LJ, Lee WL, Byrne JH. 1998. Cellular correlates of long-term sensitization in Aplysia. J Neurosci 18: 5988-5998.

Cohen TE, Kaplan SW, Kandel ER, Hawkins RD. 1997. A simplified preparation for relating cellular events to behavior: mechanisms contributing to habituation, dishabituation, and sensitization of the Aplysia gill-withdrawal reflex. J Neurosci 17: 2886-2899.

Cropper EC, Evans CG, Hurwitz I, Jing J, Proekt A, Romero A, Rosen SC. 2004. Feeding neural networks in the mollusc Aplysia. Neurosignals 13: 70-86.

Crossley M, Staras K, Kemenes G. 2016. A two-neuron system for adaptive goal-directed decision-making in Lymnaea. Nat Commun 7: 11793.

Dale N, Kandel ER, Schacher S. 1987. Serotonin produces long-term changes in the excitability of Aplysia sensory neurons in culture that depend on new protein synthesis. J Neurosci 7: 2232-2238.

Dickinson K, Wainwright ML, Mozzachiodi R. 2015. Change in excitability of a putative decision-making neuron in Aplysia serves as a mechanism in the decision not feed following food satiation. Behav Brain Res 281: 131-136.

Farruggella J, Acebo J, Wainwright M, Mozzachiodi R. 2015. Role of the nitric oxide signaling cascade in the induction of the behavioral changes produced by aversive stimuli in Aplysia. Program \# 629.16. Neuroscience Abstracts. Chicago, IL: Society for Neuroscience, 2015. Online.

Frost L, Kaplan SW, Cohen TE, Henzi V, Kandel ER, Hawkins RD. 1997. A simplified preparation for relating cellular events to behavior: contribution of LE and unidentified siphon sensory neurons to mediation and habituation of the Aplysia gill- and siphon-withdrawal reflex. J Neurosci 17: 2900-2913.

Gardner D. 1977. Interconnections of identified multiaction interneurons in buccal ganglia of Aplysia. J Neurophysiol 40: 349-361.

Glanzman DL, Mackey SL, Hawkins RD, Dyke AM, Lloyd PE, Kandel ER. 1989. Depletion of serotonin in the nervous system of Aplysia reduces the behavioral enhancement of gill withdrawal as well as the heterosynaptic facilitation produced by tail shock. J Neurosci 9: $4200-4213$.

Goldner A, Wainwright M, Mozzachiodi R. 2016. cGMP induces a decrease in the intrinsic excitability of Aplysia neuron B51 similar to that caused by sensitization training. Program \# 642.13. Neuroscience Abstracts. San Diego, CA: Society for Neuroscience, 2016. Online.

Goldsmith JR, Byrne JH. 1989. Long-term (24 hr) enhancement of the sensory-motor connection mediating tail withdrawal reflex in Aplysia is produced by nerve stimulation, an in vitro analogue of sensitization training. Soc Neurosci Abstr 15, 1283, 1989.

Hawkins RD, Abrams TW, Carew TJ, Kandel ER. 1983. A cellular mechanism of classical conditioning in Aplysia: activity-dependent amplification of presynaptic facilitation. Science 219: 400-405.

Hening WA, Walters ET, Carew TJ, Kandel ER. 1979. Motorneuronal control of locomotion in Aplysia. Brain Res 179: 231-253.

Hernandez JS, Wainwright ML, Mozzachiodi R. 2017. Long-term sensitization training in Aplysia decreases the excitability of a decision-making neuron through a sodium-dependent mechanism. Learn Mem 24: 257-261.

Jing J, Weiss KR. 2001. Neural mechanisms of motor program switch in Aplysia. J Neurosci 21: 7349-7362.

Kabotyanski EA, Baxter DA, Cushman SJ, Byrne JH. 2000. Modulation of fictive feeding by dopamine and serotonin in Aplysia. J Neurophysiol 83: 374-392.

Kandel ER. 2001. The molecular biology of memory storage: a dialog between genes and synapses. Science 294: 1030-1038.

Keifer J, Zheng Z. 2010. AMPA receptor trafficking and learning. Eur J Neurosci 32: 269-277.

Kemenes G, Staras K, Benjamin PR. 1997. In vitro appetitive classical conditioning of the feeding response in the pond snail Lymnaea stagnalis. J Neurophysiol 78: 2351-2362.

Kovac MP, Davis WJ. 1977. Behavioral choice: neural mechanisms in Pleurobranchaea. Science 198: 632-634.

Lechner HA, Baxter DA, Byrne JH. 2000. Classical conditioning of feeding behavior in Aplysia: II. Neurophysiological correlates. J Neurosci 20: 3377-3386.
Levenson J, Byrne JH, Eskin A. 1999. Levels of serotonin in the hemolymph of Aplysia are modulated by light/dark cycles and sensitization training. $J$ Neurosci 19: 8094-8103.

Levenson J, Endo S, Kategaya LS, Fernandez RI, Brabham DG, Chin J, Byrne JH, Eskin A. 2000. Long-term regulation of neuronal high-affinity glutamate and glutamine uptake in Aplysia. Proc Natl Acad Sci 97: $12858-12863$.

Lewin MR, Walters ET. 1999. Cyclic GMP pathway is critical for inducing long-term sensitization of nociceptive sensory neuron. Nat Neurosci $\mathbf{2}$ : $18-23$.

Liao X, Brou CG, Walters ET. 1999. Limited contributions of serotonin to long-term hyperexcitability of Aplysia sensory neurons. J Neurophysiol 82: 3223-3235.

Liu RY, Cleary LJ, Byrne JH. 2011. The requirement for enhanced CREB1 expression in consolidation of long-term synaptic facilitation and long-term excitability in sensory neurons of Aplysia. J Neurosci 31: 6871-6879.

Lorenzetti FD, Mozzachiodi R, Baxter DA, Byrne JH. 2006. Classical and operant conditioning differentially modify the intrinsic properties of an identified neuron. Nat Neurosci 9: 17-19.

Lukowiak K, Sahley CL. 1981. The in vitro classical conditioning of the gill withdrawal reflex of Aplysia californica. Science 212: 1516-1518.

Marder E, Bucher D, Schulz DJ, Taylor AL. 2005. Invertebrate central pattern generation moves along. Curr Biol 15: R685-R699.

Marinesco S, Carew TJ. 2002. Serotonin release evoked by tail nerve stimulation in the CNS of Aplysia: characterization and relationship to heterosynaptic plasticity. J Neurosci 22: 2299-2312.

Marinesco S, Kolkman KE, Carew TJ. 2004. Serotonergic modulation in Aplysia. I. Distributed serotonergic network persistently activated by sensitizing stimuli. J Neurophysiol 92: 2468-2486.

Mentel T, Cangiano L, Grillner S, Büschges A. 2008. Neuronal substrates for state-dependent changes in coordination between motoneuron pools during fictive locomotion in the lamprey spinal cord. J Neurosci 28: 868-879.

Mozzachiodi R, Byrne JH. 2010. More than synaptic plasticity: role of nonsynaptic plasticity in learning and memory. Trends Neurosci 33: $17-26$.

Mozzachiodi R, Lechner HA, Baxter DA, Byrne JH. 2003. In vitro analogue of classical conditioning of feeding behavior in Aplysia. Learn Mem 10: 478-494.

Mozzachiodi R, Lorenzetti FD, Baxter DA, Byrne JH. 2008. Changes in neuronal excitability serve as a mechanism of long-term memory for operant conditioning. Nat Neurosci 11: 1146-1148.

Mozzachiodi R, Baxter DA, Byrne JH. 2013. Comparison of operant and classical conditioning of feeding behavior in Aplysia. In Invertebrate learning and memory (ed. Menzel R, Benjamin P), pp. 183-193. Academic Press, London, UK.

Nargeot R, Simmers J. 2012. Functional organization and adaptability of a decision-making network in Aplysia. Front Neurosci 6: 113

Nargeot R, Baxter DA, Byrne JH. 1997. Contingent-dependent enhancement of rhythmic motor pattern: an in vitro analog of operant conditioning. J Neurosci 17: 8093-8105.

Nargeot R, Baxter DA, Byrne JH. 1999a. In vitro analog of operant conditioning in Aplysia. I. Contingent reinforcement modifies the functional dynamics of an identified neuron. J Neurosci 19: 2247-2260.

Nargeot R, Baxter DA, Byrne JH. 1999b. In vitro analog of operant conditioning in Aplysia. II. Modifications of the functional dynamics of an identified neuron contribute to motor pattern selection. J Neurosci 19: 2261-2272.

Nargeot R, Baxter DA, Byrne JH. 2002. Correlation between activity in neuron B52 and two features of fictive feeding in Aplysia. Neurosci Lett 328: $85-88$.

Noel F, Scholz KP, Eskin A, Byrne JH. 1991. Common set of proteins in Aplysia sensory neurons affected by an in vitro analogue of long-term sensitization training, 5-HT and cAMP. Brain Res 568: 67-75.

Philips GT, Sherff CM, Menges SA, Carew TJ. 2011. The tail-elicited tail withdrawal reflex of Aplysia is mediated centrally at tail sensory-motor synapses and exhibits sensitization across multiple temporal domains. Learn Mem 18: 272-282.

Plummer MR, Kirk MD. 1990. Premotor neurons B51 and B52 in the buccal ganglia of Aplysia californica: synaptic connections, effects on ongoing motor rhythms, and peptide modulation. J Neurophysiol 63: $539-558$.

Proekt A, Brezina V, Weiss KR. 2004. Dynamical basis of intentions and expectations in a simple neuronal network. Proc Natl Acad Sci 101: 9447-9452.

Sasaki K, Cropper EC, Weiss KR, Jing J. 2013. Functional differentiation of a population of electrically coupled heterogeneous elements in a microcircuit. J Neurosci 33: 93-105.

Scholz KP, Byrne JH. 1987. Long-term sensitization in Aplysia: biophysical correlates in tail sensory neurons. Science 235: 685-687. 
In vitro learning correlates in multiple circuits

Scuri R, Mozzachiodi R, Brunelli M. 2002. Activity-dependent increase of the AHP amplitude in T sensory neurons of the leech. J Neurophysiol 88: 2490-2500.

Sherff CM, Carew TJ. 2004. Parallel somatic and synaptic processing in the induction of intermediate-term and long-term synaptic facilitation in Aplysia. Proc Natl Acad Sci 101: 7463-7468.

Shields-Johnson M, Hernandez JS, Torno C, Adams KM, Wainwright M, Mozzachiodi R. 2013. Effects of Aversive stimuli beyond defensive neural circuits: Reduced excitability in an identified neuron critical for feeding in Aplysia. Learn Mem 20: 1-5.

Sieling F, Bédécarrats A, Simmers J, Prinz AA, Nargeot R. 2014. Differential roles of nonsynaptic and synaptic plasticity in operant reward learning-induced compulsive behavior. Curr Biol 24: 941-950.

Susswein AJ, Chiel HJ. 2012. Nitric oxide as a regulator of behavior: new ideas from Aplysia feeding. Prog Neurobiol 97: 304-317.

Suvrathan A, Payne HL, Raymond JL. 2016. Timing rules for synaptic plasticity matched to behavioral function. Neuron 92: 959-967.

Trudeau LE, Castellucci VF. 1993. Sensitization of the gill and siphon withdrawal reflex of Aplysia: multiple sites of change in the neuronal network. J Neurophysiol 70: 1210-1220.
Walters ET, Byrne JH. 1983. Associative conditioning of single sensory neurons suggests a cellular mechanism for learning. Science 219: 405-408.

Walters ET, Byrne JH, Carew TJ, Kandel ER. 1983a. Mechanoafferent neurons innervating tail of Aplysia. I. Response properties and synaptic connections. J Neurophysiol 50: 1522-1542.

Walters ET, Byrne JH, Carew TJ, Kandel ER. 1983b. Mechanoafferent neurons innervating tail of Aplysia. II. Response properties and synaptic connections. J Neurophysiol 50: 1522-1542.

Walters ET, Bodnarova M, Billy AJ, Dulin MF, Díaz-Ríos M, Miller MW, Moroz LL. 2004. Somatotopic organization and functional properties of mechanosensory neurons expressing sensorin-A mRNA in Aplysia californica. J Comp Neurol 471: 219-240.

Warman EN, Chiel HJ. 1995. A new technique for chronic single-unit extracellular recording in freely behaving animals using pipette electrodes. J Neurosci Methods 57: 161-169.

Zhang F, Goldsmith JR, Byrne JH. 1994. Neural analogue of long-term sensitization training produces long-term $(24$ and $48 \mathrm{~h}$ ) facilitation of the sensory-to-motor neuron connection in Aplysia. J Neurophysiol 72: $778-784$.

Received February 5, 2017; accepted in revised form May 12, 2017. 


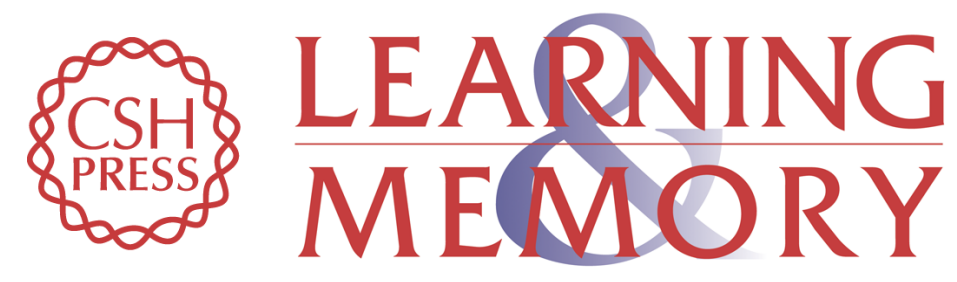

\section{A novel in vitro analog expressing learning-induced cellular correlates in distinct neural circuits}

Harris A. Weisz, Marcy L. Wainwright and Riccardo Mozzachiodi

Learn. Mem. 2017, 24:

Access the most recent version at doi:10.1101/Im.045229.117

\begin{aligned} & \hline References $\begin{array}{l}\text { This article cites } 73 \text { articles, } 39 \text { of which can be accessed free at: } \\ \text { http://learnmem.cshlp.org/content/24/8/331.full.html\#ref-list-1 }\end{array} \\ & \begin{array}{r}\text { Creative } \\ \text { Commons } \\ \text { License }\end{array} \begin{array}{l}\text { This article is distributed exclusively by Cold Spring Harbor Laboratory Press for the } \\ \text { first } 12 \text { months after the full-issue publication date (see } \\ \text { http://learnmem.cshlp.org/site/misc/terms.xhtml). After } 12 \text { months, it is available under } \\ \text { a Creative Commons License (Attribution-NonCommercial } 4.0 \text { International), as } \\ \text { described at http://creativecommons.org/licenses/by-nc/4.0/. }\end{array} \\ & \begin{array}{c}\text { Receive free email alerts when new articles cite this article - sign up in the box at the } \\ \text { top right corner of the article or click here. }\end{array} \\ & \begin{array}{l}\text { Service } \\ \text { terting }\end{array}\end{aligned}$ 